Original Research Paper

\title{
Oscillatory Behavior of Euler Cauchy Equation with Heaviside Step Function of a Bulge Function Over Various Time Domain
}

\author{
${ }^{1}$ Jitendra Kumar Pati, ${ }^{2}$ Dillip Kumar Bhukta and ${ }^{3}$ Prashanta Kumar Panda \\ ${ }^{1}$ Department of Math, C.V. Raman Global University, Bhubaneswar, Odisha, India \\ ${ }^{2}$ Department of Math, Institute of Higher Secondary Education, ITER, SOA, University Bhubaneswar, Odisha, India \\ ${ }^{3}$ Department of Mathematics, Netaji Subash Chandra Bose, Govt. College, Sambalpur, Odisha, India
}

Article history

Received: 29-01-2021

Revised: 03-03-2021

Accepted: 08-03-2021

Corresponding Author: Jitendra Kumar Pati Department of Math, C.V. Raman Global University, Bhubaneswar, Odisha, India Email: jkpati2015@gmail.com

\section{Introduction}

As we know, Laplace transform is devised to solve linear ordinary differential equations with constant coefficient as well as variable coefficients. Here we apply the Laplace transform method to solve nonhomogeneous third order differential equation with right side as a step function called as bulge function.

The Heaviside step function, or the unit step function, usually denoted by $H$ is a step function, named after Oliver Heaviside (1850-1925), the value of which is zero for negative arguments and one for positive arguments.

The function defined as follows known as Heaviside step function of a bulge function:

$$
f(t)= \begin{cases}e^{-\frac{(t-l)^{2}}{2}} & \text { if } 0<t<\xi_{1} \\ a & \text { if } t>\xi_{1}\end{cases}
$$

The step function of a bulge function is usually known as Heaviside step function of a Bulge function.

Using unit step function, it is defined as:

$$
f(t)=e^{-\frac{(t-l)^{2}}{2}}+a u\left(t-\xi_{1}\right)-e^{-\frac{(t-l)^{2}}{2}} u\left(t-\xi_{1}\right)
$$

where, $a, \xi_{1}$ are constants.

From (Yousef et al., 2019), the Laplace transform function $f(t)$ is given by:

$$
M+\frac{e^{-\xi_{1} s}}{s}+K
$$

Where:

$$
\begin{gathered}
M=e^{\frac{-l^{2}}{2}}\left[\frac{1}{s}+\frac{-1+l^{2}}{2}+\frac{l\left(s^{2}-3+l^{2}\right)}{s^{4}}\right] \\
K=A \frac{e^{-\xi_{1} s}}{s}+A l e^{-\xi_{1} s}\left[\frac{1}{s^{2}}+\frac{\xi_{1}}{s}\right]+A B e^{-\xi_{1} s}\left[\frac{2}{s^{3}}+\frac{2 \xi_{1}}{s^{2}}+\frac{\xi_{1}^{2}}{s}\right] \\
+A C e^{-\xi_{1} s}\left[\frac{6}{s^{4}}+\frac{6 \xi_{1}}{s^{3}}+\frac{3 \xi_{1}^{2}}{s^{2}}+\frac{\xi_{1}}{s}\right] \\
\text { where, } A=e^{\frac{-l^{2}}{2}}, B=\left(-\frac{1}{2}+\frac{l^{2}}{2}\right), C=\left(-\frac{1}{2}+\frac{l^{3}}{6}\right) .
\end{gathered}
$$

The Euler-Cauchy Equation

An equation of the form:

$$
a_{n} t^{n} \frac{d^{n} y}{d t^{n}}+a_{n-1} t^{n-1} \frac{d^{n-1}}{d t^{n-1}}+------+a_{1} t \frac{d y}{d t}+a_{0} y=0
$$

Here our aim is to discuss regarding solution of nonhomogeneous third order differential equation as Euler Cauchy Equation with Heaviside step function of a bulge function: 


$$
t^{3} y^{\prime \prime \prime}+a t^{2} y^{\prime \prime}+b t y^{\prime}+c y=f(t)
$$

where, $f(t)$ is defined in (1.1).

\section{Preliminary Notes}

Here we introduce some lemmas without proof whose results are useful in further section.

\section{Lemma 2.1}

The Laplace transform of the bulge function $e^{\frac{-(t-l)^{2}}{2}}$ is expressed by:

$$
L\left(e^{\frac{-(t-l)^{2}}{2}}\right)=e^{-\frac{l^{2}}{2}}\left[\frac{1}{s}+\frac{-1+l^{2}}{s^{3}}+\frac{l\left(s^{2}-3+l^{2}\right)}{s^{4}}\right]
$$

\section{Lemma 2.2}

The Laplace transform of $e^{\frac{-(t-l)^{2}}{2}} u\left(t-\xi_{1}\right)$ is expressed by:

$$
\begin{aligned}
& L\left[e^{\frac{-(t-l)^{2}}{2}} u\left(t-\xi_{1}\right)\right]=A \frac{e^{-\xi_{1} s}}{s}+A l e^{-\xi_{1} s}\left[\frac{1}{s^{2}}+\frac{\xi_{1}}{s}\right] \\
& +A B e^{-\xi_{1} s}\left[\frac{2}{s^{3}}+\frac{2 \xi_{1}}{s^{2}}+\frac{\xi_{1}^{2}}{s}\right]+A C e^{-\xi_{1} s}\left[\frac{6}{s^{4}}+\frac{6 \xi_{1}}{s^{3}}+\frac{3 \xi_{1}^{2}}{s^{2}}+\frac{\xi_{1}}{s}\right]
\end{aligned}
$$

where, $A=e^{\frac{-(t-l)^{2}}{2}}, B=\frac{-1}{2}+\frac{l^{2}}{2}, C=-\frac{1}{2}+\frac{l^{3}}{6}$.

\section{Lemma 2.3}

The Laplace transform of function $f(t)$ as in (1.1) is given by $M+\frac{a e^{-\xi_{1} s}}{s}+K$, where a, $\xi_{1}$ are constants.

\section{Main Result}

Here the Non Homogenous third order Euler Cauchy equation with Heaviside step function of a Bulge function is solved.

\section{Theorem 3.1}

The solution of the nonhomogeneous Euler Cauchy equation of the form:

$$
t^{3} y^{\prime \prime \prime}+a t^{2} y^{\prime \prime}+b t y^{\prime}+c y=f(t)= \begin{cases}e^{-\frac{(t-l)^{2}}{2}} & \text { if } 0<t<\xi_{1} \\ a & \text { if } t>\xi_{1}\end{cases}
$$

With $y(0)=H, y^{\prime}(0)=E, y^{\prime \prime}(0)=F$ is expressed by:
$y=-\frac{\phi_{3}}{b}+P e^{\frac{a}{2 D} t} \cos \psi_{1} t+Q e^{\frac{a}{2 D} t} \sin \psi_{1} t$

$+e^{\frac{l^{2}}{2}}\left[t\left(\frac{1}{b}+---\right)+t^{2}\left\{\frac{\left(-1+l^{2}\right) a D^{2}}{2 b H_{1}}+-----\right\}\right]$

$+R(\delta(t)+---)+S\left(t-\xi_{1}\right)+u\left(t-\xi_{1}\right)+T\left(t-\xi_{1}\right)^{2} u\left(t-\xi_{1}\right)$

$+V\left(t-\xi_{1}\right)^{3} u\left(t-\xi_{1}\right)+e^{\frac{a}{2 D}\left(t-\xi_{1}\right)} \cos \psi_{1}\left(t-\xi_{1}\right) u\left(t-\xi_{1}\right) W$

$+e^{\frac{a}{2 D}\left(t-\xi_{1}\right)} \sin \psi_{1}\left(t-\xi_{1}\right) u\left(t-\xi_{1}\right) N$

Where:

$$
\begin{aligned}
P & =\left[-\frac{a D^{2} e^{\frac{-l^{2}}{2}}}{b H_{1}}+\frac{\phi_{3} D^{3}}{b}+---\right] \\
Q & =\left[\frac{\phi_{3} D^{2} a}{2 b \psi_{1}}+\frac{a^{2} D e^{-\frac{l^{2}}{2}}}{2 b}\right] \\
R & =e^{-\frac{l^{2}}{2}} \frac{4 D^{3}}{b a}\left(\frac{a^{2}-D H_{1}}{H_{1}}\right) \\
S & =\left[\left(\frac{A}{b}+\frac{A l}{b}\right)+----\right] \\
T & =\left[\frac{A l}{2 b}+\frac{A B \xi_{1}}{b}+---\right] \\
V & =\left[\frac{A B}{3 b}+---\right] \\
W & =\left[-\frac{a}{b \psi_{1}} D^{3}+---\right] \\
N & =\left[-\frac{a^{2} D^{2}}{2 \psi_{1}}-\frac{A l D^{3} \xi_{1}}{b \psi_{1}}+---\right]
\end{aligned}
$$

\section{Proof}

Now the given equation is:

$$
t^{3} y^{\prime \prime \prime}+a t^{2} y^{\prime \prime}+b t y^{\prime}+c y=f(t),
$$

With initial conditions $y(0)=H, y^{\prime}(0)=E, y^{\prime \prime}(0)=F$. Taking Laplace transform both sides, we get:

$$
\begin{aligned}
& -\frac{d^{3}}{d s^{3}}\left(s^{3} y(s)-s^{2} y(0)-s y^{\prime}(0)-y^{\prime \prime}(0)\right) \\
& +a \frac{d^{2}}{d s^{2}}\left(s^{2} y(s)-s y(0)-y^{\prime}(0)\right) \\
& -b(s y(s)-y(0))+c y(s)=L\{f(t)\}
\end{aligned}
$$

Using $D=\frac{d}{d s}$ and boundary conditions $y(0)=H, y^{\prime}(0)$ $=E, y^{\prime \prime}(0)=F$, we get: 


$$
\begin{aligned}
& y(s)\left(-D^{3} s^{3}+a D^{2} s^{2}-b s+c\right)=-D^{3} s^{2} H-D^{3} s E-D^{3} F+a H D^{2} s+E a D^{2}-b H+M+\frac{a e^{-\xi_{1} s}}{s}+K \\
& \Rightarrow y(s)=\frac{-D^{3} s^{2} H+\left(-D^{3} E+a H D^{2}\right) s+\left(-D^{3} F+E a D^{2}-b H\right)+M+\frac{a e^{-\xi_{1} s}}{s}+K}{\left(-D^{3} s^{3}+a D^{2} s^{2}-b s+c\right)}
\end{aligned}
$$

Letting $\phi_{1}=-D^{3} s^{2} H, \phi_{2}=-D^{3} E+a H D^{2}, \phi_{3}=-D^{3} F+E a D^{2}-b H$

So:

$$
y(s)=\frac{\phi_{1} s^{2}+\phi_{2} s+\phi_{3}+M+\frac{a e^{-\xi_{1} s}}{s}+K}{-D^{3} s^{3}+a D^{2} s^{2}-b s}
$$

for taking $c=0$ in particular.

Now:

$\frac{\phi_{1} s^{2}}{-D^{3} s^{3}+a D^{2} s^{2}-b s}=\frac{\phi_{1} s}{\left(s-\frac{a}{2 D}\right)^{2}+\psi_{1}}$

where, $\psi_{1}=\frac{-D a^{2}+4 b}{4 D^{3}}$

Similarly, we have:

$\frac{\phi_{2} s}{-D^{3} s^{3}+a D^{2} s^{2}-b s}=\frac{-\phi_{2}}{\left(s-\frac{a}{2 D}\right)^{2}+\psi_{1}}$

$\frac{\phi_{3}}{-D^{3} s^{3}+a D^{2} s^{2}-b s}=\frac{-\phi_{3}}{s\left(D^{3} s^{2}-a D^{2} s+b\right)}$

$=\frac{A}{s}+\frac{B s+c}{D^{3} s^{2}-a D^{2} s+b}$

Hence:

$\frac{\phi_{3}}{-D^{3} s^{3}+a D^{2} s^{2}-b s}=\frac{\phi_{3}}{b s}+\frac{\frac{\phi_{3} D^{3} s}{b}-\frac{\phi_{3} a D^{2}}{b}}{\left(s-\frac{a}{2 D}\right)^{2}+\psi_{1}}$

In continuing:

$$
\begin{aligned}
& \frac{M}{-D^{3} s^{3}+a D^{2} s^{2}-b s} \\
& =e^{\frac{-l^{2}}{2}}\left[\frac{1}{s}+\frac{-1+l^{2}}{2}+\frac{l\left(s^{2}-3+l^{2}\right)}{s^{4}}\right]\left(\frac{1}{b s}+\frac{\frac{-D^{3} s}{b}+\frac{a D^{2}}{b}}{\left(s-\frac{a}{2 D}\right)^{2}+\psi_{1}}\right) \\
& \left.=e^{\frac{-l^{2}}{2}}\left[\frac{1}{b s^{2}}+\frac{\frac{-D^{3}}{b}}{\left(s-\frac{a}{2 D}\right)^{2}+\psi_{1}}+\frac{\frac{a D^{2}}{b}}{\left.s\left(s-\frac{a}{2 D}\right)^{2}+\psi_{1}\right]}\right]\right)
\end{aligned}
$$

$$
\begin{aligned}
& +e^{\frac{-l^{2}}{2}}\left(\frac{-1+l^{2}}{b s^{4}}+\frac{\left(-1+l^{2}\right)\left[\frac{-D^{3}}{b}+\frac{a D^{2}}{b}\right]}{s^{2}\left[\left(s-\frac{a}{2 D}\right)^{2}+\psi_{1}\right]}+\frac{\frac{a D^{2}}{b}\left(-1+l^{2}\right)}{s^{3}\left[\left(s-\frac{a}{2 D}\right)^{2}+\psi_{1}\right]}\right) \\
& +e^{\frac{-l^{2}}{2}}\left(\frac{l\left(s^{2}-3+l^{2}\right)}{b s^{5}}+\frac{l\left(s^{2}-3+l^{2}\right)\left(-\frac{D^{3}}{b}\right)}{s^{3}\left[\left(s-\frac{a}{2 D}\right)^{2}+\psi_{1}\right]}+\frac{\frac{l}{b} a D^{2}\left(s^{2}-3+l^{2}\right)}{s^{4}\left[\left(s-\frac{a}{2 D}\right)^{2}+\psi_{1}\right]}\right)
\end{aligned}
$$

Now:

$$
\begin{aligned}
& \frac{1}{s\left[\left(s-\frac{a}{2 D}\right)^{2}+\psi_{1}\right]}=\frac{A}{s}+\frac{B s+C}{\left[\left(s-\frac{a}{2 D}\right)^{2}+\psi_{1}\right]} \\
& =\frac{1}{s\left[\psi_{1}+\frac{a^{2}}{4 D^{2}}\right]}+\frac{\psi_{1}+\frac{a^{2}}{4 D^{2}}}{\left[\left(s-\frac{a}{2 D}\right)^{2}+\psi_{1}\right]}+\frac{\frac{a}{4 D^{2}}+D \psi_{1}}{\left[\left(s-\frac{a}{2 D}\right)^{2}+\psi_{1}\right]}
\end{aligned}
$$

And:

$\frac{1}{s^{2}\left[\left(s-\frac{a}{2 D}\right)^{2}+\psi_{1}\right]}=\frac{A s+B}{s^{2}}+\frac{C s+E}{s^{2}+\frac{a^{2}}{4 D^{2}}-\frac{s a}{D}+\psi_{1}}$

$=\frac{a}{s\left(\psi_{1}+\frac{a^{2}}{4 D^{2}}\right)^{2}}+\frac{1}{s^{2}\left(\psi_{1}+\frac{a^{2}}{4 D^{2}}\right)}+\frac{\frac{-a s}{\left(\psi_{1}+\frac{a^{2}}{4 D^{2}}\right)^{2}}}{\left(s-\frac{a}{2 D}\right)^{2}+\psi_{1}}+\frac{\frac{a^{2}-\frac{a^{2}}{4 D}-D \psi_{1}}{\left(\psi_{1}+\frac{a^{2}}{4 D^{2}}\right)^{2}}}{\left(s-\frac{a}{2 D}\right)^{2}+\psi_{1}}$

Similarly:

$$
\begin{aligned}
& \frac{1}{s^{3}\left[\left(s-\frac{a}{2 D}\right)^{2}+\psi_{1}\right]}=\frac{A}{s}+\frac{B}{s^{2}}+\frac{C}{s^{3}}+\frac{J s+E}{\left(s-\frac{a}{2 D}\right)^{2}+\psi_{1}} \\
& =\frac{\frac{\left(D-a^{2}\right.}{\left(\psi_{1}+\frac{a^{2}}{4 D^{2}}\right)^{2}}+\frac{a}{s}+\frac{D\left(\psi_{1}+\frac{a^{2}}{4 D^{2}}\right)^{2}}{s^{2}}+\frac{1}{\psi_{1}+\frac{a^{2}}{4 D^{2}}}}{s^{3}} \\
& +\frac{\frac{\left(a^{2}-D\right) s}{\left(\psi_{1}+\frac{a^{2}}{4 D^{2}}\right)^{2}}+\frac{-a\left(a^{2}+\frac{a^{2}}{4 D^{2}}+\psi_{1}-D\right)}{D\left(\psi_{1}+\frac{a^{2}}{4 D^{2}}\right)^{2}}}{\left(s-\frac{a}{2 D}\right)^{2}+\psi_{1}}
\end{aligned}
$$


Continuing, we have:

$$
\begin{aligned}
& \frac{1}{s^{4}\left[\left(s-\frac{a}{2 D}\right)^{2}+\psi_{1}\right]}=\frac{A}{s}+\frac{B}{s^{2}}+\frac{C}{s^{3}}+\frac{J}{s^{4}}+\frac{N s+P}{\left(s-\frac{a}{2 D}\right)^{2}+\psi_{1}}
\end{aligned}
$$

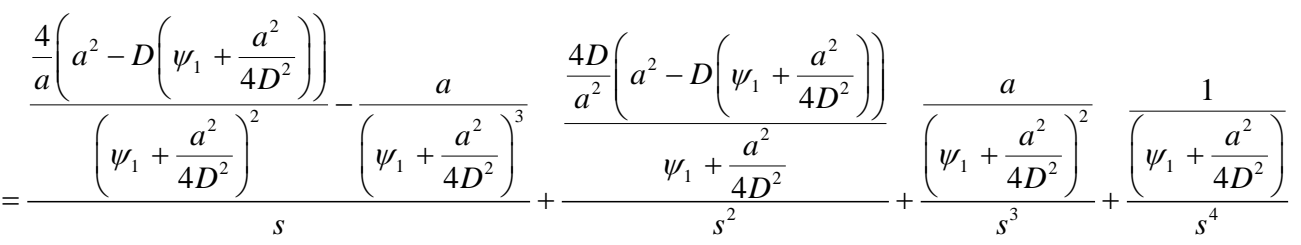

$$
\begin{aligned}
& +\frac{\frac{a^{2}}{\left(\psi_{1}+\frac{a^{2}}{4 D^{2}}\right)^{3}}-\frac{4\left[\left(a^{2}-D\left(\psi_{1}+\frac{a^{2}}{4 D^{2}}\right)\right] s\right.}{a\left(\psi_{1}+\frac{a^{2}}{4 D^{2}}\right)^{2}}+\frac{-8 D}{\psi_{1}+\frac{a^{2}}{4 D^{2}}}+\frac{4 D^{2}}{a^{2}}+\frac{4 a^{2}}{\left(\psi_{1}+\frac{a^{2}}{4 D^{2}}\right)^{2}}-\frac{1}{\left(\psi_{1}+\frac{a^{2}}{4 D^{2}}\right)^{3}}}{\left(s-\frac{a}{2 D}\right)^{2}+\psi_{1}}
\end{aligned}
$$

So, letting $\left(\psi_{1}+\frac{a^{2}}{4 D^{2}}\right)=H_{1}$, we have:

$\frac{M}{-D^{3} s^{3}+a D^{2} s^{2}-b s}=e^{\frac{-l^{2}}{2}}\left[\frac{1}{b s^{2}}+\frac{\frac{-D^{3}}{b}}{\left(s-\frac{a}{2 D}\right)^{2}+\psi_{1}}+\frac{a D^{2}}{b}\left(\frac{a}{H_{1} s}-\frac{\frac{s}{H_{1}}+\frac{a}{D H_{1}}}{\left(s-\frac{a}{2 D}\right)^{2}+\psi_{1}}\right)\right]$

$+e^{\frac{-l^{2}}{2}}\left[\frac{\left(-1+l^{2}\right)}{b s^{4}}+\frac{\left(1-l^{2}\right) D^{3}}{b}\left\{\frac{\frac{a s}{H_{1}}+\frac{1}{H_{1}}}{s^{2}}+\frac{\frac{-a s}{H_{1}^{2}}+\frac{a^{2}+D H_{1}}{D H_{1}^{2}}}{\left(s-\frac{a}{2 D}\right)^{2}+\psi_{1}}\right\}\right]$

$+e^{\frac{-l^{2}}{2}} \frac{\left(-1+l^{2}\right) a D^{2}}{b}\left\{\frac{D-a^{2}}{H_{1}^{2} s}+\frac{a}{D H_{1} s^{2}}+\frac{1}{H_{1} s^{3}}+\frac{\frac{\left(a^{2}-D\right) s}{H_{1}^{2}}+\frac{\frac{-a}{D}\left(a^{2}+H_{1}-D\right)}{H_{1}^{2}}}{\left(s-\frac{a}{2 D}\right)^{2}+\psi_{1}}\right\}$

$+e^{\frac{-l^{2}}{2}}\left[\frac{l\left(s^{2}-3+l^{2}\right)}{b s^{5}}-\frac{l\left(s^{2}-3+l^{2}\right) D^{3}}{b}\left\{\frac{D-a^{2}}{H_{1}^{2} s}+\frac{a}{D H_{1}^{2} s^{2}}+\frac{1}{H_{1} s^{3}}+\frac{\frac{\left(a^{2}-D\right) s}{H_{1}^{2}}+\frac{a\left(-a^{2}+D-1\right)}{D H_{1}^{2}}}{\left(s-\frac{a}{2 D}\right)^{2}+\psi_{1}}\right\}\right]$

$+e^{\frac{-l^{2}}{2}}\left[\frac{l}{b} a D^{2}\left(s^{2}-3+l^{2}\right)\right]\left\{\frac{\frac{4\left(a^{2}-D H_{1}\right)}{a H_{1}^{2}}-\frac{a}{H_{1}^{3}}}{s}+\frac{\frac{4 D\left(a^{2}-D H_{1}\right)}{a^{2} H_{1}}}{s^{2}}+\frac{a}{H_{1}^{2} s^{3}}+\frac{1}{H_{1} s^{4}}\right\}$

$+e^{\frac{-l^{2}}{2}} \frac{l}{b} a D^{2}\left(s^{2}-3+l^{2}\right)\left[\frac{s\left(\frac{1}{H_{1}^{3}}-\frac{4\left(a^{2}-D H_{1}\right)}{a H_{1}^{2}}\right)+\frac{-8 D}{H_{1}}+\frac{4 D^{2}}{a^{2}}+\frac{4 a^{2}}{H_{1}^{2}}-\frac{1}{H_{1}^{3}}}{\left(s-\frac{a}{2 D}\right)^{2}+\psi_{1}}\right]$ 
So:

$$
\begin{aligned}
& \frac{a e^{-\xi_{1} s}}{s\left(-D^{3} s^{3}+a D^{2} s^{2}-b s\right)}=\frac{a e^{-\xi_{1} s}}{s}\left[\frac{1}{b s}+\frac{\frac{-D^{3} s+a D^{2}}{b}}{\left(s-\frac{a}{2 D}\right)^{2}+\psi_{1}}\right] \\
& =\frac{a e^{-\xi_{1} s}}{b s^{2}}+\frac{-a e^{-\xi_{1} s} D^{3}}{b\left[\left(s-\frac{a}{2 D}\right)^{2}+\psi_{1}\right]}+\frac{a^{2} D^{2} e^{-\xi_{1} s}}{b s\left[\left(s-\frac{a}{2 D}\right)^{2}+\psi_{1}\right]}
\end{aligned}
$$

Similarly:

$$
\begin{aligned}
& \frac{K}{-D^{3} s^{3}+a D^{2} s^{2}-b s}=\left\{A \frac{e^{-\xi_{1} s}}{s}+A l e^{-\xi_{1} s}\left[\frac{1}{s^{2}}+\frac{\xi_{1}}{s}\right]+A B e^{-\xi_{1} s}\left[\frac{2}{s^{3}}+\frac{2 \xi_{1}}{s^{2}}+\frac{\xi_{1}^{2}}{s}\right]\right. \\
& \left.+A C e^{-\xi_{1} s}\left[\frac{6}{s^{4}}+\frac{6 \xi_{1}}{s^{3}}+\frac{3 \xi_{1}^{2}}{s^{2}}+\frac{\xi_{1}}{s}\right]\right\}\left[\frac{1}{b s}+\frac{\frac{-D^{3} s+a D^{2}}{b}}{\left(s-\frac{a}{2 D}\right)^{2}+\psi_{1}}\right] \\
& =\frac{A e^{-\xi_{1} s}}{b s^{2}}+\frac{A e^{-\xi_{1} s} s}{s}\left[\frac{\frac{-D^{3} s+a D^{2}}{b}}{\left(s-\frac{a}{2 D}\right)^{2}+\psi_{1}}\right]+A l e^{-\xi_{1} s}\left[\frac{1}{s^{2}}+\frac{\xi_{1}}{s}\right]\left[\frac{1}{b s}+\frac{\frac{-D^{3} s+a D^{2}}{b}}{\left(s-\frac{a}{2 D}\right)^{2}+\psi_{1}}\right] \\
& +A B e^{-\xi_{1} s}\left[\frac{2}{s^{3}}+\frac{2 \xi_{1}}{s^{2}}+\frac{\xi_{1}^{2}}{s}\right]\left[\frac{1}{b s}+\frac{\frac{-D^{3} s+a D^{2}}{b}}{\left(s-\frac{a}{2 D}\right)^{2}+\psi_{1}}\right] \\
& +A C e^{-\xi_{1} s}\left[\frac{6}{s^{4}}+\frac{6 \xi_{1}}{s^{3}}+\frac{3 \xi_{1}^{2}}{s^{2}}+\frac{\xi_{1}}{s}\right]\left[\frac{1}{b s}+\frac{\frac{-D^{3} s+a D^{2}}{b}}{\left(s-\frac{a}{2 D}\right)^{2}+\psi_{1}}\right]
\end{aligned}
$$

Using (3.1) and inverse Laplace transform, we have:

$$
y_{1}(s)=\frac{\phi_{1} s^{2}}{-D^{3} s^{3}+a D^{2} s^{2}=b s}=\frac{\phi_{1}\left(s-\frac{a}{2 D}+\frac{a}{2 D}\right)}{\left(s-\frac{a}{2 D}\right)^{2}+\psi_{1}}
$$

Where, $\psi_{1}=\frac{-D a^{2}+4 b}{4 D^{3}}$.

So:

$$
y_{1}(t)=-\phi_{1} e^{\frac{a}{2 D} t}\left[\cos \psi_{1} t+\frac{a}{2 D \psi_{1} t} \sin \psi_{1} t\right]
$$

From (3.2) and inverse Laplace Transform, we have:

$$
y_{2}(s)=\frac{\phi_{2} s}{-D^{3} s^{3}+a D^{2} s^{2}-b s}=\frac{-\phi_{2}}{\left(s-\frac{a}{2 D}\right)^{2}+\psi_{1}}
$$

So:

$$
y_{2}(t)=\frac{\phi_{2}}{\psi_{1}} e^{\frac{a}{2 D} t} \sin \psi_{1} t
$$

From (3.3) and inverse Laplace Transform, we have:

$$
y_{3}(s)=\frac{\phi_{3}}{-D^{3} s^{3}+a D^{2} s^{2}-b s}=\frac{-\phi_{3}}{b s}+\frac{\frac{\phi_{3} D^{3} s}{b}-\frac{\phi_{3} a D^{2}}{b}}{\left(s-\frac{a}{2 D}\right)^{2}+\psi_{1}}
$$

So:

$y_{3}(t)=\frac{-\phi_{3}}{b}+\frac{\phi_{3} D^{3}\left(s-\frac{a}{2 D}+\frac{a}{2 D}\right)}{\left(s-\frac{a}{2 D}\right)^{2}+\psi_{1}}-\frac{\phi_{3} a D^{2}}{b \psi_{1}}+e^{\frac{a}{2 D} t} \sin \psi_{1} t$

$=\frac{-\phi_{3}}{b}+\frac{\phi_{3} D^{3}}{b} e^{\frac{a}{2 D}}\left\{\cos \psi_{1} t+\frac{a}{2 D \psi_{1}} \sin \psi_{1} t\right\}$ 
From (3.4) we have:

$$
\begin{aligned}
& y_{4}(s)=\frac{M}{-D^{3} s^{3}+a D^{2} s^{2}-b s}=A_{1}(s)+B_{1}(s) \\
& +C_{1}(s)+D_{1}(s)+E_{1}(s)+F_{1}(s)
\end{aligned}
$$

So, using inverse Laplace transform we obtain:

$$
\begin{aligned}
& y_{4}(t)=A_{1}(t)+B_{1}(t)+C_{1}(t)+D_{1}(t)+E_{1}(t)+F_{1}(t) \\
& A_{1}(t)=e^{\frac{-l^{2}}{2}}\left\{\frac{t}{b}-\frac{D^{3}}{b \psi_{1}} \operatorname{sni} \psi_{1} t+\frac{a D^{2}}{2}\left[\frac{1}{H_{1}}-\frac{1}{H_{1}} e^{\frac{a}{2 D} t} \cos \psi_{1} t+\left(\frac{-a}{2 D}+\frac{a}{D H_{1}}\right) \frac{1}{\psi_{1}} e^{\frac{a}{2 D} t} \sin \psi_{1} t\right]\right\} \\
& B_{1}(t)=e^{\frac{-l^{2}}{2}}\left\{\frac{\left(-1+l^{2}\right)}{6 b}+\frac{\left(1-l^{2}\right) D^{3}}{b}\left(\frac{a}{H_{1}}+\frac{1}{H_{1}} t+\frac{-a}{H_{1}^{2}} e^{\frac{a}{2 D} t} \cos \psi_{1} t\right)+\left(\frac{-a^{2}}{2 D H_{1}^{2}}+\frac{a^{2}+D H_{1}}{D H_{1}^{2}}\right) \frac{1}{\psi_{1}} e^{\frac{a}{2 D} t} \sin \psi_{1} t\right\} \\
& C_{1}(t)=e^{\frac{-l^{2}}{2}} \frac{\left(-1+l^{2}\right) a D^{2}}{b}\left\{\frac{D-a^{2}}{H_{1}^{2}}+\frac{a}{D H_{1}} t+\frac{t^{2}}{2 H_{1}}+\frac{a^{2}-D}{H_{1}^{2}} e^{\frac{a}{2 D} t} \cos \psi_{1} t\right. \\
& \left.+\left(\frac{a}{2 D}\left[\frac{a^{2}-D}{H_{1}^{2}}\right]-\frac{a}{D} \frac{a^{2}+H_{1}-D}{H_{1}^{2}}\right) \frac{1}{\psi_{1}} e^{\frac{a}{2 D} t} \sin \psi_{1} t\right\} \\
& D_{1}(t)=e^{\frac{-l^{2}}{2}}\left[\frac{l}{b}\left(\frac{t^{2}}{2}-\frac{3 t^{4}}{24}+\frac{l^{2} t^{4}}{24 b}\right)-\frac{a l D^{2}}{H_{1}^{2}}\left(\delta(t)-\left(3+l^{2}\right) l\right)+------\right] \\
& E_{1}(t)+F_{1}(t)=e^{\frac{-l^{2}}{2}}\left[\frac{4 D^{3} l\left(a^{2}-D H_{1}\right)}{b a H_{1}}\left(\delta(t)-\left(3+l^{2}\right) t\right)+\frac{l a D^{2}\left(\frac{-8 D}{H_{1}}+\frac{4 D^{2}}{a^{2}}+\frac{4 a^{2}}{H_{1}^{2}}-\frac{1}{H_{1}^{3}}\right)}{\psi_{1}} e^{\frac{a}{2 D} t} \sin \psi_{1} t+---\right]
\end{aligned}
$$

From (3.5) we have:

$$
y_{5}(s)=\frac{a e^{-\xi_{1} s}}{s\left(-D^{3} s^{3}+a D^{2} s^{2}-b s\right)}=\frac{a e^{-\xi_{1} s}}{b s^{2}}+\frac{-a D^{3} e^{-\xi_{1} s}}{b\left[\left(s-\frac{a}{2 D}\right)^{2}+\psi_{1}\right]}+\frac{a^{2} D^{2} e^{-\xi_{1} s}}{b s\left[\left(s-\frac{a}{2 D}\right)^{2}+\psi_{1}\right]}
$$

So:

$$
y_{5}(t)=\frac{a}{b}\left(t-\xi_{1}\right) U\left(t-\xi_{1}\right)-\frac{a D^{3}}{b \psi_{1}} e^{\frac{a\left(t-\xi_{1}\right)}{2 D}} \cos \psi_{1}\left(t-\xi_{1}\right) U\left(t-\xi_{1}\right)-\frac{a^{2} D^{2}}{2 \psi_{1}} e^{\frac{a\left(t-\xi_{1}\right)}{2 D}} \sin \psi_{1}\left(t-\xi_{1}\right) U\left(t-\xi_{1}\right)+----
$$

From (3.6) we have:

$$
y_{6}(s)=\frac{K}{-D^{3} s^{3}+a D^{2} s^{2}-b s}
$$

So:

$$
\begin{aligned}
& y_{6}(t)=\frac{A}{b}\left(t-\xi_{1}\right) U\left(t-\xi_{1}\right)-\frac{A D^{3}}{b \psi_{1}} e^{\frac{a\left(t-\xi_{1}\right)}{2 D}} \sin \psi_{1}\left(t-\xi_{1}\right) U\left(t-\xi_{1}\right)+\frac{A a D^{2}}{b} L^{-1}\left\{\frac{e^{-\xi_{1} s}}{\left[s\left[\left(s-\frac{a}{2 D}\right)^{2}+\psi_{1}\right]\right.}\right\} \\
& +\frac{A l}{2 b}\left(t-\xi_{1}\right)^{2} U\left(t-\xi_{1}\right)+\frac{A l}{b}\left(t-\xi_{1}\right) U\left(t-\xi_{1}\right)-\frac{A l D^{3}}{b} L^{-1}\left\{\frac{e^{-\xi_{1} s}}{\left[\left(s-\frac{a}{2 D}\right)^{2}+\psi_{1}\right.}\right]
\end{aligned}
$$




$$
\begin{aligned}
& -\frac{A l D^{3} \xi_{1}}{b \psi_{1}} e^{\frac{a\left(t-\xi_{1}\right)}{2 D}} \sin \psi_{1}\left(t-\xi_{1}\right) U\left(t-\xi_{1}\right)+\frac{A l a D^{2}}{b} L^{-1}\left\{\frac{e^{-\xi_{1} s}\left(\frac{1}{s^{2}}+\frac{\xi_{1}}{s}\right)}{\left(s-\frac{a}{2 D}\right)^{2}+\psi_{1}}\right\} \\
& +\frac{A B}{3 b}\left(t-\xi_{1}\right)^{3} U\left(t-\xi_{1}\right)+\frac{A B \xi_{1}}{b}\left(t-\xi_{1}\right)^{2} U\left(t-\xi_{1}\right)+\frac{A B \xi_{1}^{2}}{b}\left(t-\xi_{1}\right) U\left(t-\xi_{1}\right) \\
& +L^{-1}\left\{A B e^{-\xi_{1} s}\left[\frac{2}{s^{3}}+\frac{2 \xi_{1}}{s^{2}}+\frac{\xi_{1}^{2}}{s}\right]\left[\frac{\frac{-D^{3} s-a D^{2}}{b}}{\left(s-\frac{a}{2 D}\right)^{2}+\psi_{1}}\right]\right\} \\
& +L^{-1}\left\{A C e^{-\xi_{1} s}\left[\frac{6}{s^{4}}+\frac{6 \xi_{1}}{s^{3}}+\frac{3 \xi_{1}^{2}}{s^{2}}+\frac{\xi_{1}}{s}\right]\left[\frac{1}{b s}+\frac{\frac{-D^{3} s+a D^{2}}{b}}{\left(s-\frac{a}{2 D}\right)^{2}+\psi_{1}}\right]\right\}
\end{aligned}
$$

In simplifying we have:

$$
\begin{aligned}
& y_{6}(t)=\left(t-\xi_{1}\right) U\left(t-\xi_{1}\right)\left[\frac{A}{b}+\frac{A l}{b}+----\right]+\left(t-\xi_{1}\right)^{2} U\left(t-\xi_{1}\right)\left[\frac{A l}{2 b}+\frac{A B \xi_{1}}{b}+----\right] \\
& +\left(t-\xi_{1}\right)^{3} U\left(t-\xi_{1}\right)\left[\frac{A B}{3 b}+------\right]+e^{\frac{a\left(t-\xi_{1}\right)}{2 D}} \sin \psi_{1}\left(t-\xi_{1}\right) U\left(t-\xi_{1}\right)\left[\frac{A D^{3}}{b \psi_{1}}+----\right] \\
& +e^{\frac{a\left(t-\xi_{1}\right)}{2 D}} \cos \psi_{1}\left(t-\xi_{1}\right) U\left(t-\xi_{1}\right)\left[\frac{-A l D^{3}}{b \psi_{1} H_{1}}+----\right]
\end{aligned}
$$

So the final solution is:

$$
\begin{aligned}
& y(t)=y_{1}(t)+y_{2}(t)+y_{3}(t)+y_{4}(t)+y_{5}(t)+y_{6}(t) \\
& =\frac{-\phi_{3}}{b}+e^{\frac{a}{2 D} t} \cos \psi_{1} t\left[\frac{-a D^{2} e^{-\frac{l^{2}}{2}}}{b H_{1}}+\frac{\phi_{3} D^{3}}{b}+---\right]+e^{\frac{a}{2 D} t} \sin \psi_{1} t\left[\frac{\phi_{2} D^{2} a}{2 b \psi_{1}}+\frac{a^{2} D e^{\frac{-l^{2}}{2}}}{2 b}+---\right] \\
& +e^{\frac{-l^{2}}{2}}\left[t\left(\frac{1}{b}+-----\right)+t^{2}\left(\frac{\left(-1+l^{2}\right) a D^{2}}{2 b H_{1}}+----\right)\right] \\
& +e^{\frac{-l^{2}}{2}} \frac{4 D^{3}}{b a}\left(\frac{a^{2}-D H_{1}}{H_{1}}\right)(\delta(t)+---) \\
& +\left(t-\xi_{1}\right) U\left(t-\xi_{1}\right)\left[\left(\frac{A}{b}+\frac{a}{b}\right)+-----\right]+\left(t-\xi_{1}\right)^{2} U\left(t-\xi_{1}\right)\left[\frac{A l}{2 b}+\frac{A B \xi_{1}}{b}+--\right] \\
& +\left(t-\xi_{1}\right)^{3} U\left(t-\xi_{1}\right)\left[\frac{A B}{3 b}+----\right]+e^{\frac{a\left(t-\xi_{1}\right)}{2 D}} \cos \psi_{1}\left(t-\xi_{1}\right) U\left(t-\xi_{1}\right)\left[-\frac{a D^{3}}{b \psi_{1}}+----\right] \\
& +e^{\frac{a\left(t-\xi_{1}\right)}{2 D}} \sin \psi_{1}\left(t-\xi_{1}\right) U\left(t-\xi_{1}\right)\left[-\frac{a^{2} D^{2}}{2 \psi_{1}}+\frac{A l D^{3} \xi_{1}}{b \psi_{1}}+----\right]
\end{aligned}
$$

\section{Comparative Study of Oscillatory Property of Euler Cauchy Equation}

In this section, the authors desire to have a comparative study of oscillatory property of Euler Cauchy equation with homogenous and Non homogenous output. Take the homogenous equation as:

$$
t^{3} y^{\prime \prime \prime}+t^{2} y^{\prime \prime}+t y^{\prime}+y=0, y(0)=1, y^{\prime}(0)=2, y^{\prime \prime}(0)=3
$$

The graph of the solution is in Fig. 1.

The equation seems to be oscillatory within the interval and converging towards oscillation. Going within the range $[0,200]$ in Fig. 2, we find the equation is 
oscillatory and seems to be converging towards oscillation within the $t$ value from 50 to 100 .

In discussion for a non homogenous equation, we have the Euler Cauchy equation with bulge function as:

$$
t^{3} y^{\prime \prime}+t^{2} y^{\prime \prime}+t y^{\prime}+y=e^{-\frac{(t-1)^{2}}{2}}, y(0)=1, y^{\prime}(0)=2, y^{\prime \prime}(0)=3
$$

The graph of the solution is in Fig. 3.

It seems to be oscillatory within $t$ value from 20 to 40 (refer Fig. 3).

Similarly within the range [0,200], the graph of solution is in Fig. 4. It seems to be oscillatory within $t$ value from 50 to 100 .

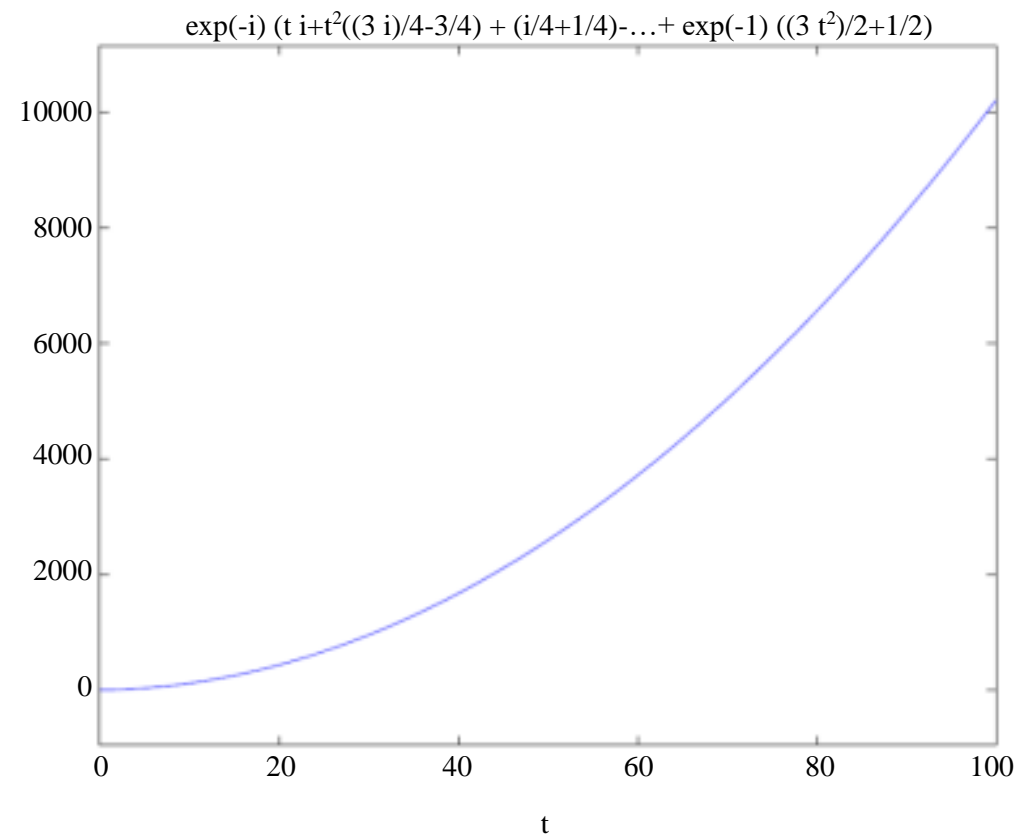

Fig. 1: Solution of Homogenous Euler Cauchy equation within the range $[0,100]$

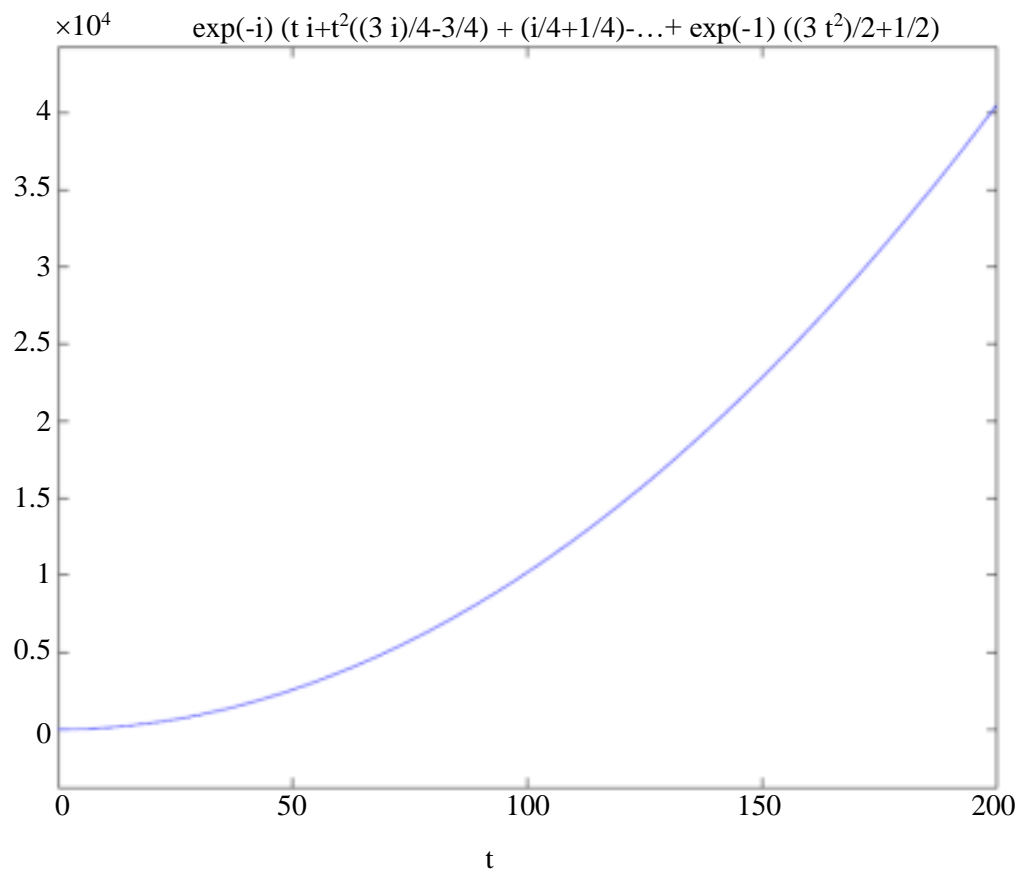

Fig. 2: Solution of Homogenous Euler Cauchy equation within the range [0,200] 


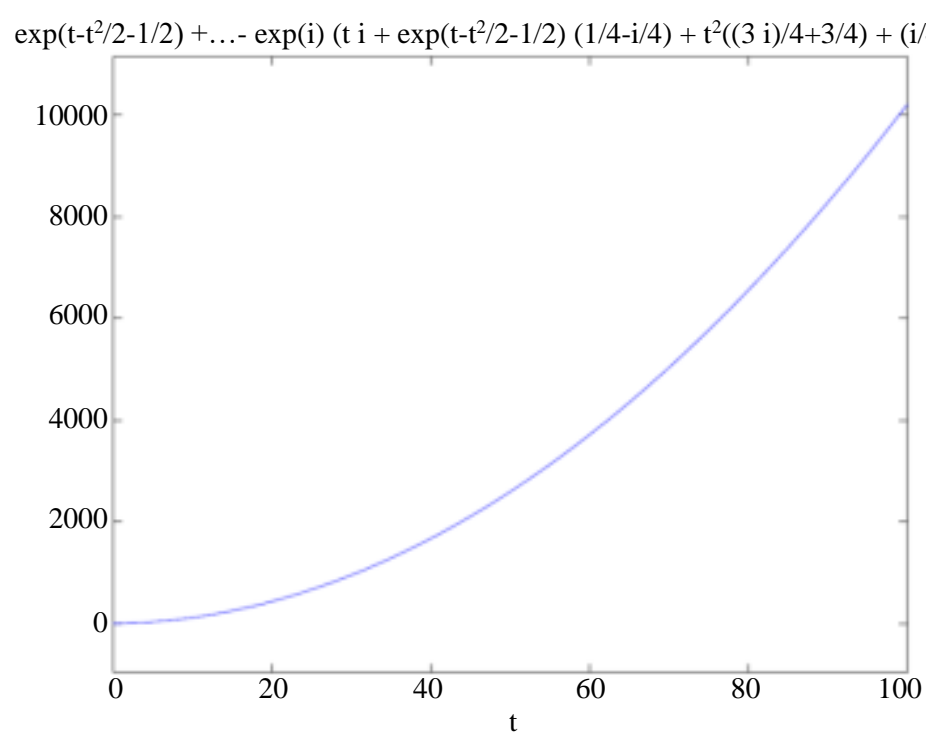

Fig. 3: Solution of Non Homogenous Euler Cauchy equation with bulge function within the range $[0,100]$

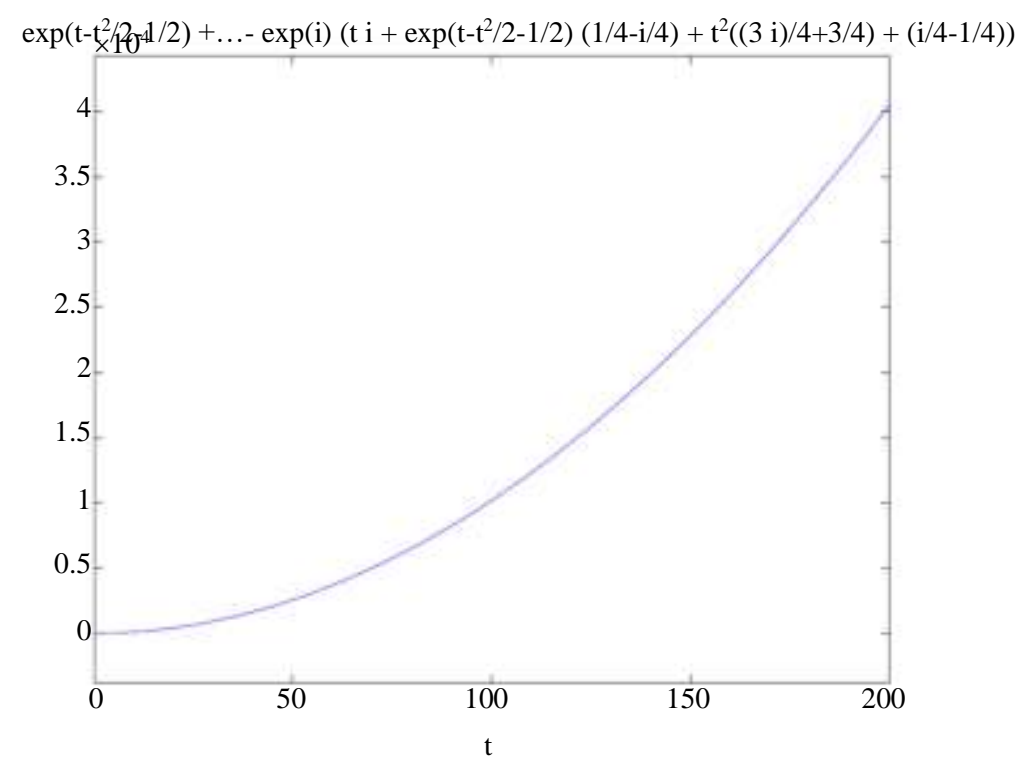

Fig. 4: Solution of Non Homogenous Euler Cauchy equation with bulge function within the range $[0,200]$

Again for discussing the non homogenous equation as Euler Cauchy equation with heaviside step function of a bulge function as:

$$
\begin{aligned}
& t^{3} y^{\prime \prime \prime}+t^{2} y^{\prime \prime}+t y^{\prime}+y=e^{-\frac{(t-1)^{2}}{2}}+U(t-\xi) \\
& -e^{-\frac{(t-1)^{2}}{2}} U(t-\xi), y(0)=1, y^{\prime}(0)=2, y^{\prime \prime}(0)=3
\end{aligned}
$$

The graph of the solution for $l=1, \xi=1$ within the range $[0,100]$ is in Fig. 5. Fig. 6.

Within the range $[0,200]$ the graph of solution is in
It seems to be oscillatory within $t$ value from 50 to 100 .

Within the range $[-5000,5000]$ the graph of solution is in Fig. 7.

It also seems to be oscillatory.

If the range is extended to $[-10,000,10,000]$, we have the graph in Fig. 8.

\section{Main Comparison}

Now using the result of (3.7), if an equation is taken to be the linear combination of all the functions as exponential, Dirac delta and Heaviside step functions, we may get one equation as: 
$y=e^{t} \cos t+e^{t} \sin t+\delta(t)+(t-1) U(t-1)$

$+(t-1)^{2} U(t-1)+e^{t-1} \cos (t-1) U(t-1)$

$+e^{t-1} \sin (t-1) U(t-1)$.

by taking $l=1, \xi=1$.
Graph of the equation is in Fig. 9.

It is strongly oscillatory within the range $[4,6]$.

Similarly within the range $[-100,100]$, the graph of equation is in Fig. 10.

It is also strongly oscillatory within the range $[0,100]$ and varies constantly towards the oscillation.

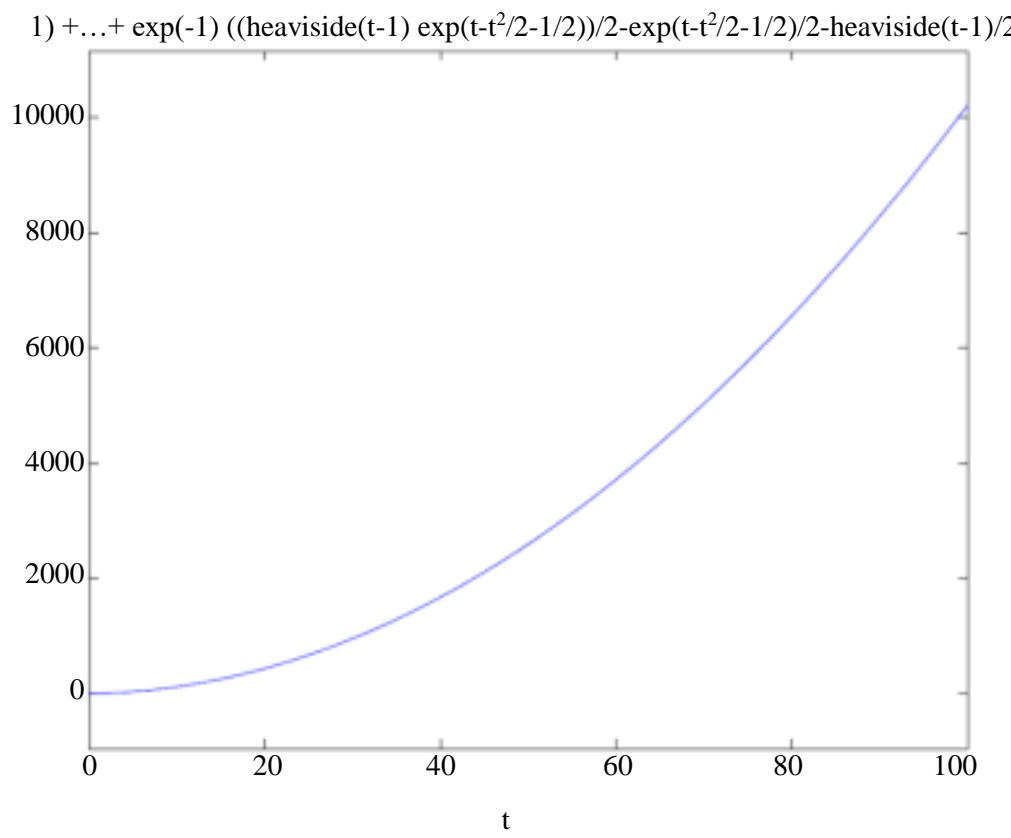

Fig. 5: Solution of Non Homogenous Euler Cauchy equation with heaviside step function of a bulge function within the range [0,100]

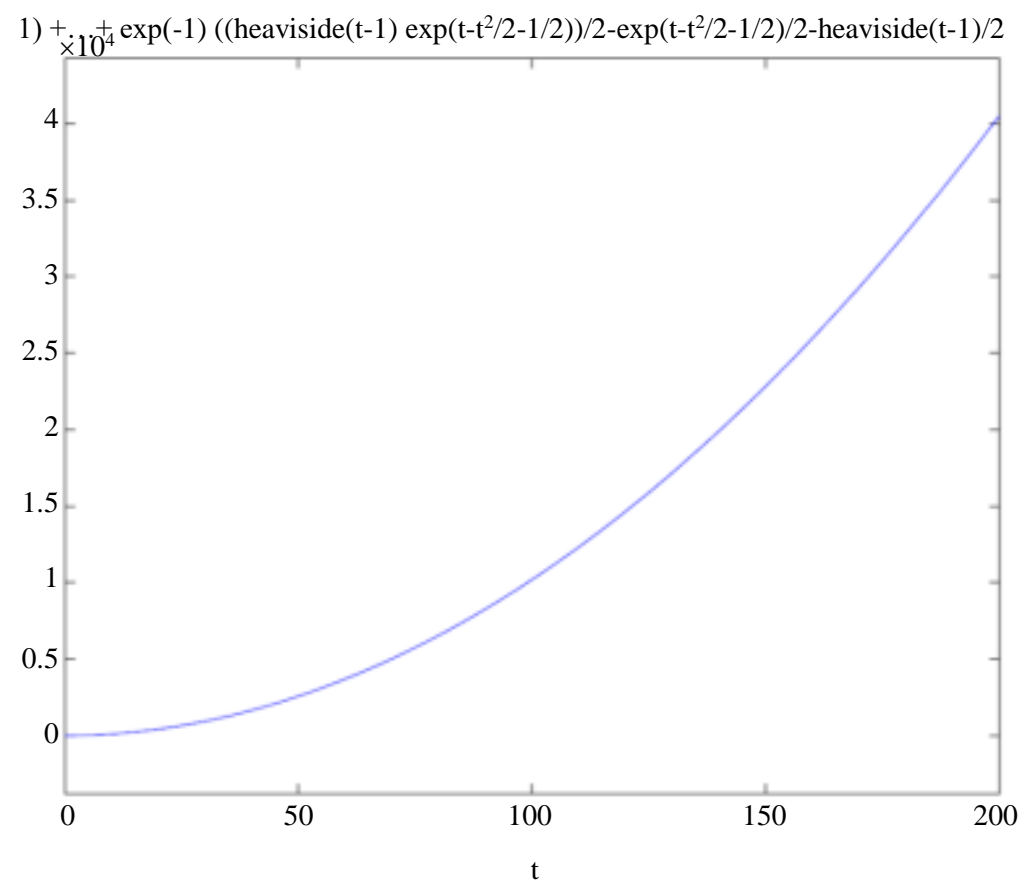

Fig. 6: Solution of Non Homogenous Euler Cauchy equation with heaviside step function of a bulge function within the range [0,200] 


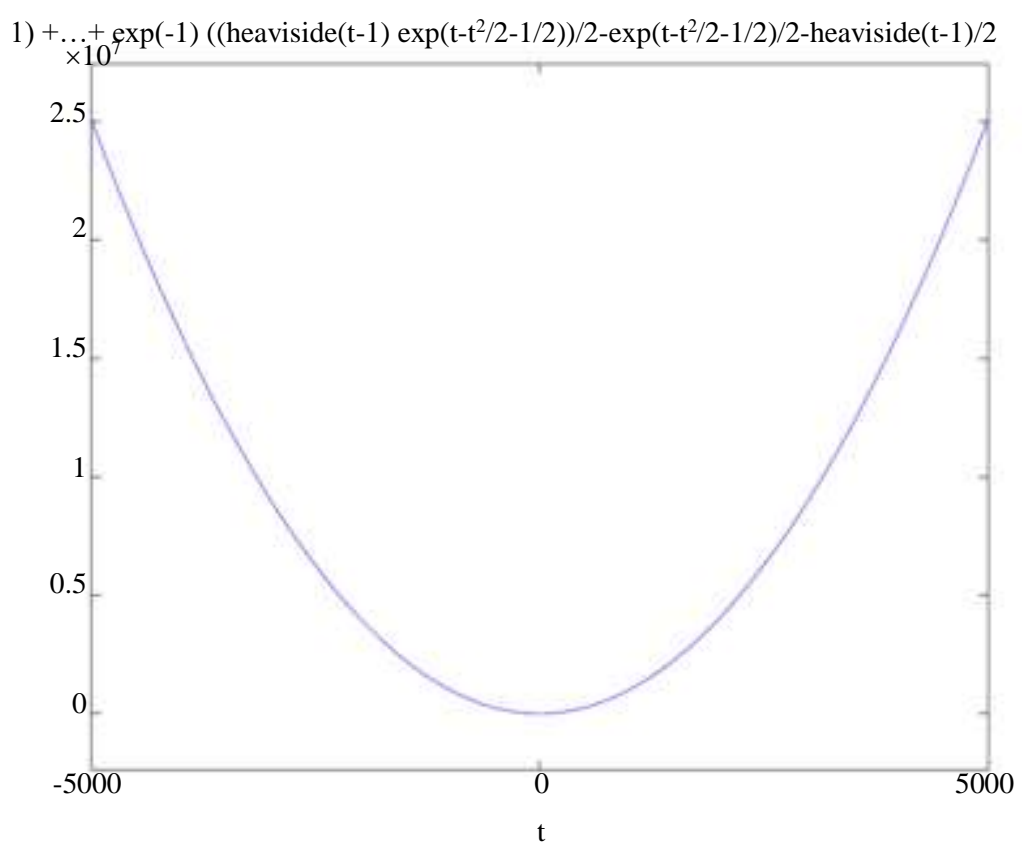

Fig. 7: Solution of Non Homogenous Euler Cauchy equation with heaviside step function of a bulge function within the range [5000,5000]

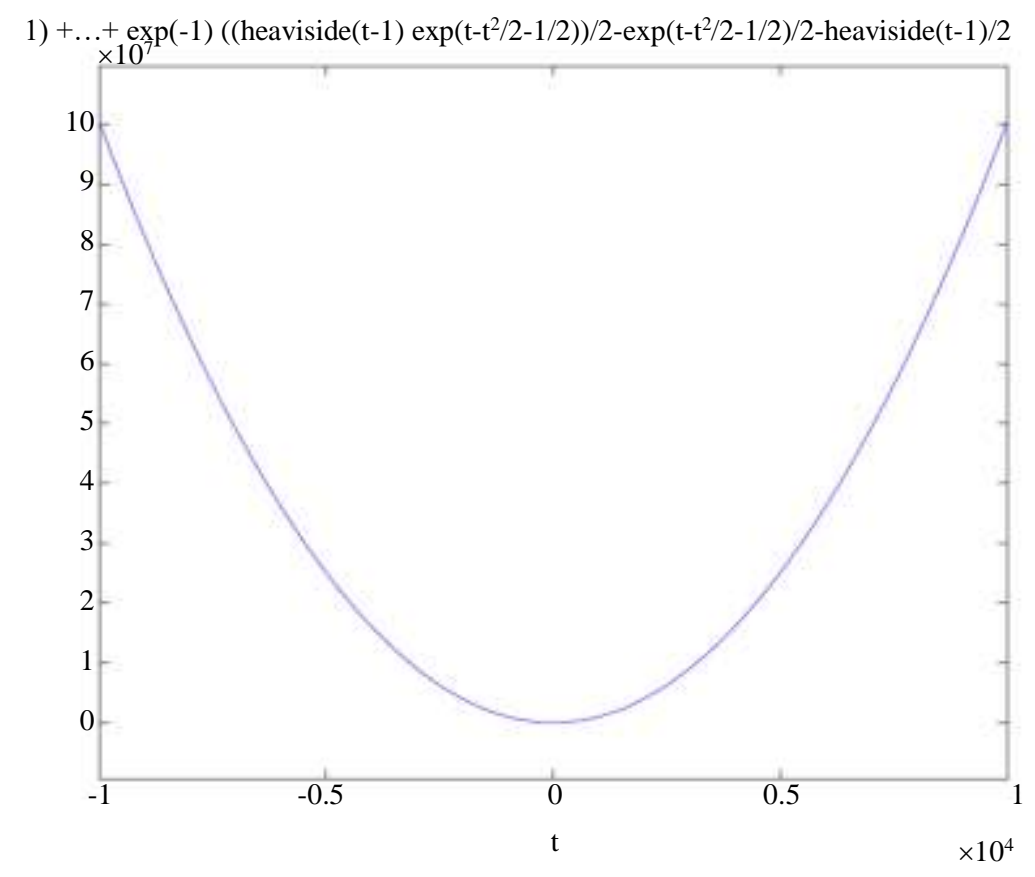

Fig. 8: Solution of Non Homogenous Euler Cauchy equation with heaviside step function of a bulge function within the range $[-10000,10000]$

Similarly within the range [-200,200], the graph of equation is in Fig. 11.

It is also oscillatory within the range $[0,150]$ and at the point $t=100$, the graph varies constantly towards the oscillation.
Similarly within the range $[-500,500]$, the graph of equation is in Fig. 12.

Here also we conclude that it is oscillatory.

Similarly within the range [200,1000] we have the graph is also oscillatory as in Fig. 13. 


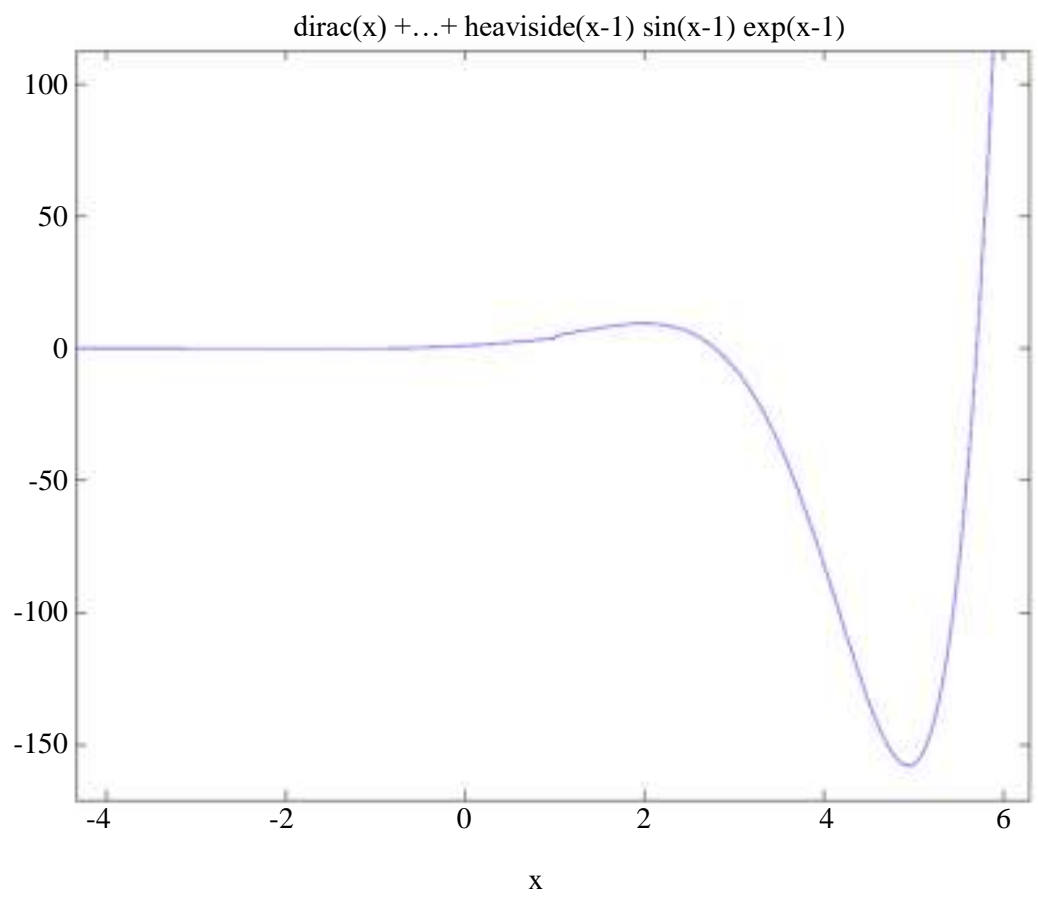

Fig. 9: Solution of the Equation (4.1) with heaviside step function, Dirac delta function within the range $[-4,6]$

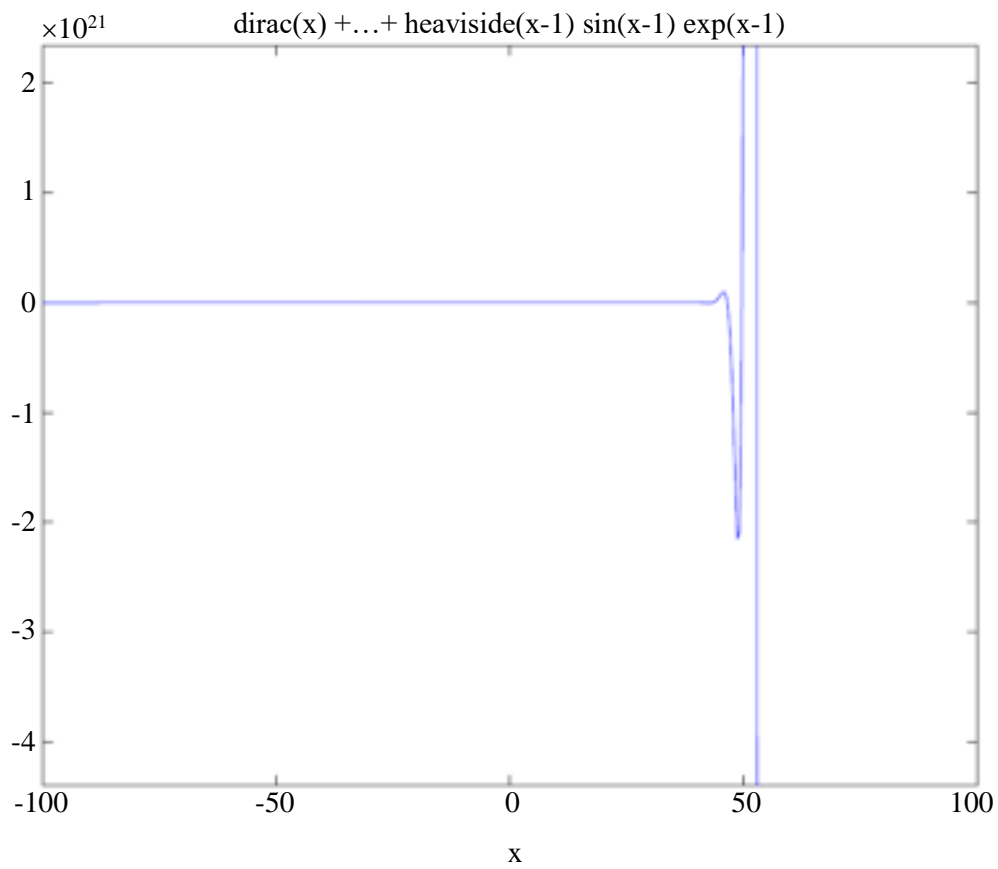

Fig. 10: Solution of an Equation (4.1) with heaviside step function, Dirac delta function within the range [-100,100]

If we compare the result (3.7) with the Equation (4.1), we find the difference up to some values of constant.

Since the Equation (4.1) is oscillatory and the Euler Cauchy equation with heaviside step function of a bulge function is also oscillatory as per the Fig. 5 to 8 , so the solution (3.7) satisfies as per the condition of oscillation.

Here the author wants to point out that the oscillatory behavior of Euler Cauchy equation is really 
good by taking the non homogenous output as heaviside step function of a bulge function, since it includes discontinuous functions like Dirac delta and unit step function. The presence of these kind of functions helps to measure the oscillatory behavior up to a period of time.

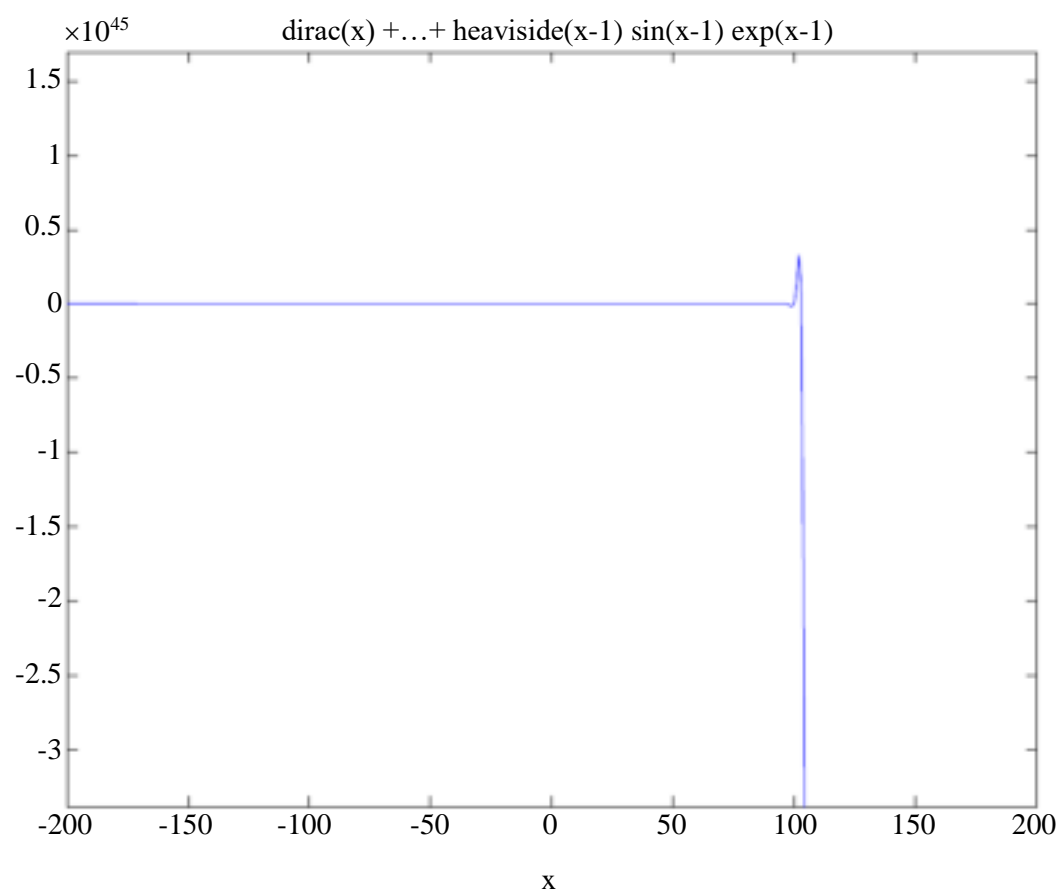

Fig. 11: Solution of an Equation (4.1) with heaviside step function, Dirac delta function within the range [-200, 200]

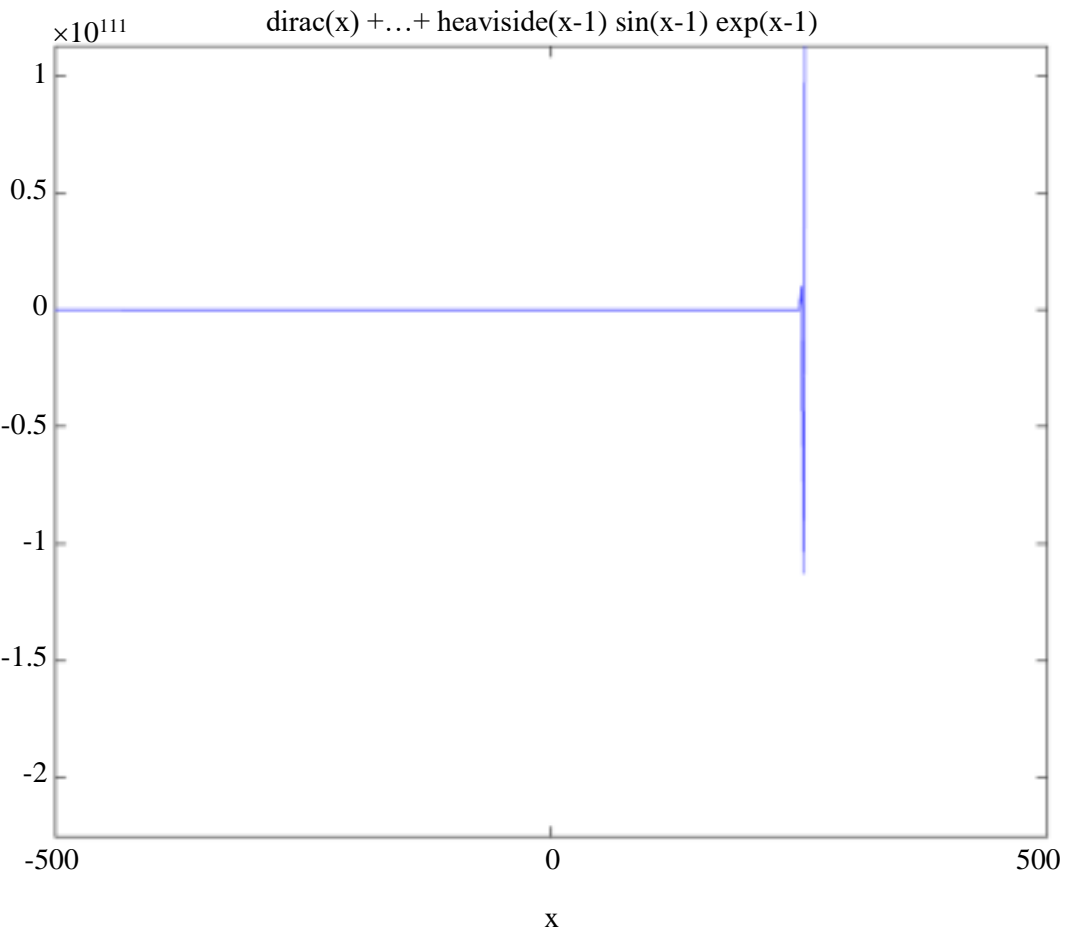

Fig. 12: Solution of an Equation (4.1) with heaviside step function, Dirac delta function within the range [-500,500] 


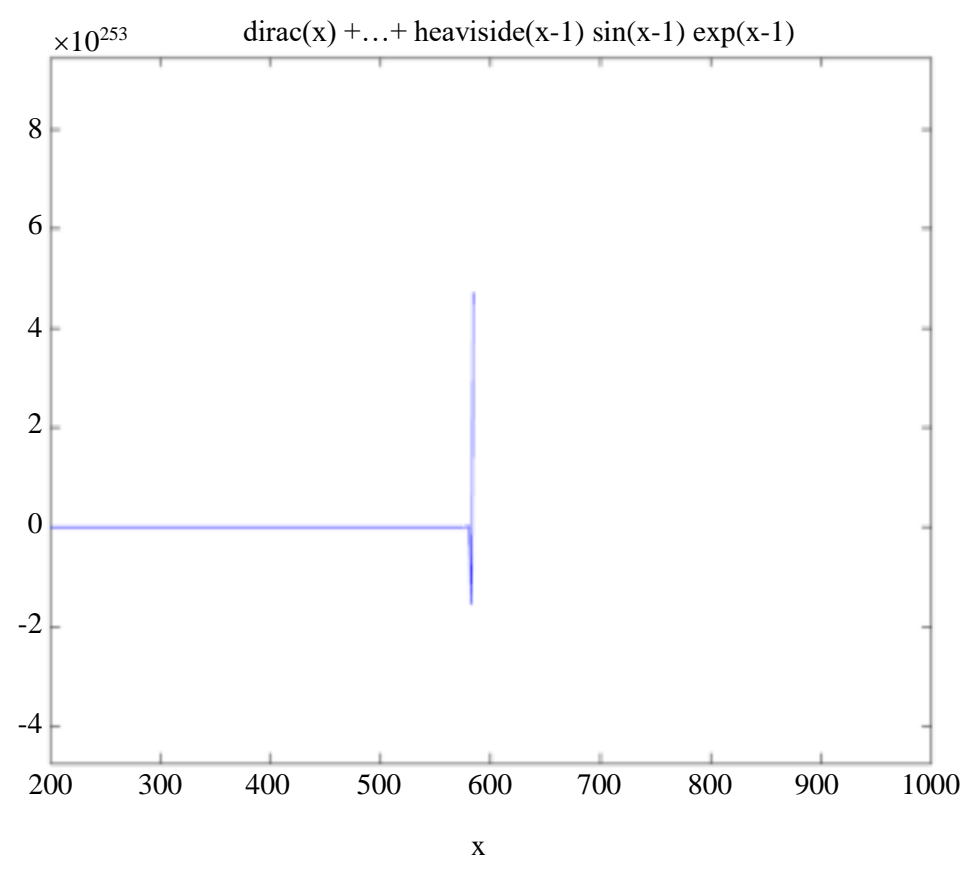

Fig. 13: Solution of an Equation (4.1) with heaviside step function, Dirac delta function within the range[200,1000]

\section{Conclusion}

In this article, authors discuss about solution of nonhomogeneous Euler Cauchy equation with heaviside step function of a bulge function in different time domain and at last there is a comparative study of oscillatory behavior of the concerned equation with homogenous and non homogenous output.

The non homogenous equation with right hand side as piecewise continuous function like heaviside step function, causes strongly oscillatory behavior in compare to homogenous output.

This process of comparative study may be applicable to check the oscillatory and non oscillatory behavior of equation concerned to mass spring system with damping and without damping. In the near future, we intend to conduct more research as a continuation of this work.

\section{Acknowledgment}

Authors are really thankful for remarkable comments from the reviewers to publish the paper

\section{Author's Contributions}

All authors equally contributed in this work.

\section{Ethics}

This is complete new work and no chance of copyrighting from any sources. There is no problem to publish the paper.

\section{References}

Yousef, F., Alquran, M., Jaradat, I., Momani, S., \& Baleanu, D. (2019). Ternary-fractional differential transform schema: theory and application. Advances in Difference Equations, 2019(1), 1-13. https://doi.org/10.1186/s13662-019-2137-x 